\title{
On the Use of Fuzzy Logic in Dependable Cloud Management
}

\author{
Sara Foresti, Vincenzo Piuri, Gerson A. Soares \\ Università degli Studi di Milano, 26013 Crema - Italy, Email: firstname.lastname @ unimi.it
}

\begin{abstract}
The effective and efficient use of dependable cloud infrastructures requires the agreement between users and cloud providers on resources, services, operating conditions, and features as well as the mapping of users' requirements onto the cloud architecture. In this paper, we identify the different ways in which fuzzy logic can be profitably adopted in performing these tasks, providing flexibility in capturing users' needs and dealing with complex architectures and conflicting or hardly-satisfiable requirements. We specifically put forward the idea of using fuzzy logic at the user-side, to enable the specification of users' needs in crisp or fuzzy ways and their homogenous processing.
\end{abstract}

Keywords-Fuzzy Logic; Dependable Cloud; Service Level Agreement; Resource Allocation

\section{INTRODUCTION}

Thanks to its elasticity, convenience, and economic advantage, cloud computing is today one of the reference paradigms for data storage and management and for running (heavy computational) applications. To fully benefit from the advantages of cloud systems, users need to have sufficient guarantees on the overall dependability of these systems, including the reliability and resilience of cloud architectures, the continuous availability of the services they pay for, the security of the operating environment and infrastructure, the protection guarantees on data and applications in the whole operating flow [1].

To ensure full user satisfaction, the user and the cloud provider need to carefully specify and agree upon a comprehensive Service Level Agreement (SLA). Such SLA should define the infrastructure configuration expected by the user to support her applications. Conventional approaches to SLA specification (e.g., [2]) allow users to define her requirements by choosing the most appropriate values for the parameters made available by the cloud provider (e.g., the amount of data storage and speed in data transfer). On the basis of the SLA agreed with the user, the cloud provider identifies a mapping of the user's needs (in terms of the minimum resources required by the user's applications) onto the cloud architecture and services. During operation, possible changes in the cloud infrastructure (e.g., faults of connections or processing nodes, and security vulnerabilities) may impair the ability of satisfying users' requirements in the due time or even at all. Monitoring and possibly dynamically adjusting the resource mapping is therefore desirable to ensure complete dependability of the cloud architecture.

While generally SLA specifications require the definition of values for configuration parameters, users would appreciate the

This work was supported in part by: EC within 7FP project ABC4EU (grant 312797); EC within H2020 project ESCUDO-CLOUD (grant 644579), Italian MIUR within PRIN project "GenData 2020" (grant 2010RTFWBH), and Brazilian CAPES Foundation (grant BX5949/13-0). availability of a more flexible way for expressing their needs, since users often do not have an exact understanding of the real requirements of their applications. Also, the management of complex and dynamic environments would benefit from flexibility in reasoning on the mappings of users' requirements onto cloud resources. Some works have started considering fuzzy logic as a convenient approach to reach such a flexibility (e.g, [3], [4], [5], [6]). In this paper, we investigate and identify different ways in which fuzzy logic can be used in the various components of dependable cloud systems (Section II). We also introduce the innovative use of fuzzy logic at the user side to support SLA specification (Section III). The use of fuzzy logic for specifying users' requirements has not yet been considered in cloud management, while it has a prominent role in modern scenarios as dependable pervasive solutions are envisioned.

\section{Fuzzy Logic in Cloud Systems Management}

Cloud systems management can take advantage of fuzzy logic in different ways, from representing knowledge and observed facts in a way similar to humans (i.e., with natural language and its imprecisions), to approximate reasoning including incomplete or inaccurate information (like humans are able to do). We identify and analyze the different uses of fuzzy logic in cloud systems management, pointing out its adoption in various components, and the advantages it provides.

Fuzzy specification of users' preferences on requirements. In many practical situations it may not be possible to satisfy all the requirements that the user specified in the SLA because they are conflicting or there are not enough resources. User's requirements do not always have the same relevance and impact on the correct and timely execution of the applications. Fuzzy logic can be used to enable users to easily define the relative relevance or their preferences among requirements. For instance, in a big data application, a user can specify that storage requirement has "high importance", application performance has "medium importance", and user interface and interaction have "low importance". Merging requirements taking into account their relative preferences may guide the resource allocation engine in finding a feasible solution, by solving a multi-objective optimization problem.

Fuzzy allocation of resources to tasks. The allocation of cloud resources to the tasks of user's applications is a complex problem, which needs to take into account various aspects (e.g., applications performance, users and provider costs, energy consumption, resource usage, security, data protection, and system reliability and availability). This problem has conflicting goals and is usually addressed using a multiobjective decision making approach. In dynamic scenarios, where tasks are continuously activated/deactivated, a strategy that reaches the global optimum at each update is not practical 
as it may imply moving tasks and data. To overcome this issue, incremental approaches allocate (as optimally as possible) only the new tasks according to their SLAs. Fuzzy logic can be useful to support flexible reasoning during mapping of the requests of new tasks onto available cloud resources, especially by merging all requirements similarly to adaptable human reasoning. In particular, fuzzy similarity can be used to measure the distance between solutions and find the optimal allocation of resources.

Fuzzy monitoring and prediction of cloud status. Cloud systems evolve mainly because of workload activation and dynamic operation changes, faults or malfunctioning components, security threats and attacks, changing application needs, and need for energy saving. Monitoring the cloud system permits to understand and possibly anticipate critical situations, ensuring dependability based on continuity and quality of service. In fact, starting from observed behavioral trends, prediction tools are able to infer the future system behavior from the past condition and current situation. In critical situations, applications reallocation may be suggested to maintain the desired level of service quality and fulfill the SLAs. Monitoring and prediction of the cloud system operation can benefit from flexible reasoning based on fuzzy logic, suited to understand the cloud status and identify (nearly-) critical situations.

Fuzzy dynamic allocation of resources to tasks. Dynamic reallocation of resources to applications tasks can be adopted in dependable cloud system management to ensure dynamic adaptation to changing operating conditions. This could in fact guarantee continuous complete fulfillment of the SLAs. The resource (re-)allocation algorithm should then be executed, either periodically or when the status monitor/predictor points out a significant change, which may impair (or is shortly expected to impair) the normal system operation. Fuzzy logic can be useful also to support flexible multi-objective reasoning for dynamic adaptation of the resource mapping to the evolving operating conditions.

\section{FUZZY USER REQUIREMENTS}

Users often tend to overestimate their requirements in SLAs, to prevent possible problems during the working of their applications. This however leads to a waste of paid resources and reduces resource availability in the cloud. To limit this waste of resources (and money for final users), we propose to use fuzzy logic to support SLA specification. Fuzzy logic allows users to define their applications needs in a flexible way, capturing natural linguistic expressions, when users are not specialists in information systems and technologies and when requirements are not so crispy defined or easily definable. Fuzzy logic can support the definition of users' requirements as fuzzy parameters and fuzzy concepts as follows.

Fuzzy parameters. Fuzzy parameters permit users to define their requirements when they are unable to determine an exact, specific value of a characteristic of the cloud environment, but they are fully conscious of the required size of the considered characteristic and are linguistically able to describe it (e.g., with adjectives of periphrases) For instance, a user who would like to use a large key for the encryption of her data, may not have a precise idea of the needed key length. In this case, the user may prefer to state that a long key is needed, accepting a conventionally defined concept of "long" as a fuzzy range of values. Mutual agreement about the significance of

\begin{tabular}{l|l} 
Fuzzy label & Range \\
\hline very short & $1-32 \mathrm{bit}$ \\
short & $16-128 \mathrm{bit}$ \\
medium & $64-256 \mathrm{bit}$ \\
long & $128-1024 \mathrm{bit}$ \\
very long & $512-2048 \mathrm{bit}$ \\
& (a)
\end{tabular}

Fuzzy concept |Parameters high data security encryption: Elliptic Curve min. key length: 384 bit redundancy: 5 copies HMAC: SHA-512

hash key length: 512 bit

(b)

Figure 1. An example of fuzzy specification of key length parameter (a) and of data security concept (b)

linguistic expressions is essential to understand and satisfy the user's requirement. A key length requirement defined in the conventional crisp way has the following typical form:

Key length $=384$ bit

and may be better replaced by a fuzzy definition like:

Key length should be "long"

provided that the user follows the fuzzy definition of sizes defined by the provider (e.g., Figure 1(a)). The separation between ranges of key length is not crisp, but ranges may overlap. This fuzzy specification of parameters in SLAs allows cloud providers to manage with higher elasticity their resources, without living them unused when applications do not explicitly demand for them. This enables for better resource allocation, with higher quality of service at lower costs for both the provider and the users.

Fuzzy concepts. Fuzzy concepts operate at abstract level, allowing users to define features that do not directly correspond to a cloud characteristic or parameter, but map on an appropriate combination of them. Fuzzy logic can provide the mathematical foundation for merging real characteristics and metrics, by translating the linguistic high-level description given by the user. For example, if a user wants to protect her data but does not know the characteristics that are relevant for data protection, she may prefer to request "high data security". The user then relies on fuzzy logic for formalizing and processing this concept, translating it into proper parameter values (e.g., see Figure 1(b)).

Fuzzy parameters and fuzzy concepts can then be transformed in a format that the cloud management engine can process in a homogeneous way with other crisp requirements, to take all of them into account in a comprehensive resource allocation strategy and optimization.

\section{REFERENCES}

[1] P. Samarati and S. De Capitani di Vimercati, "Cloud security: Issues and concerns," in Encyclopedia on Cloud Computing, S. Murugesan and I. Bojanova, Eds. Wiley, 2016 (to appear).

[2] R. Buyya, C. S. Yeo, S. Venugopal, J. Broberg, and I. Brandic, "Cloud computing and emerging IT platforms: Vision, hype, and reality for delivering computing as the 5th utility," Future Generation Computer Systems, vol. 25, no. 6, pp. 599-616, 2009.

[3] C. Anglano, M. Canonico, and M. Guazzone, "FC2Q: Exploiting fuzzy control in server consolidation for cloud applications with SLA constraints," Concurrency and Computation: Practice and Experience, vol. 22, no. 6, 2014.

[4] A. V. Dastjerdi and R. Buyya, "Compatibility-aware cloud service composition under fuzzy preferences of users," IEEE TCC, vol. 2, no. 1, pp. 1-13, 2014.

[5] S. Frey, L. Claudia, C. Reich, and N. Clarke, "Cloud QoS scaling by fuzzy logic," in IEEE IC2E, Boston, MA, March 2014.

[6] J. Rao, Y. Wei, J. Gong, and C. Z. Xu, "DynaQoS: Model-free selftuning fuzzy control of virtualized resources for QoS provisioning," in IEEE IWQoS, San Jose, CA, June 2011. 